\title{
Rhododendrin inhibits toll-like receptor-7-mediated psoriasis-like skin inflammation in mice
}

\begin{abstract}
Yoon-Jae Jeon ${ }^{1,4}$, Shyam Kishor Sah ${ }^{1,4}$, Hee Seung Yang1, Ji Hae Lee ${ }^{2}$, Jongheon Shin $^{3}$ and Tae-Yoon Kim ${ }^{1}$
Many active compounds present in Rhododendron brachycarpum have been used in traditional Oriental medicine for the treatment of various skin diseases. However, the precise mechanism of action of the compounds isolated from $R$. brachycarpum and their relevance as therapeutics for the treatment of psoriasis remain elusive. In this study, we report that rhododendrin isolated from $R$. brachycarpum strongly inhibits imiquimod (IMQ)-induced psoriasis-like skin inflammation in mice. We showed that topical treatment with rhododendrin reduces IMQ-induced skin hyperplasia, inflammatory mononuclear cell infiltration and the expression of pro-inflammatory mediators in mouse skin. In addition, we found that rhododendrin inhibits the activation of the TLR-7/NF-KB and mitogen-activated protein kinase pathways in both IMQ-induced psoriasis-like skin inflammation in mice and in normal human epidermal keratinocytes treated with IMQ. These results suggest that rhododendrin has an anti-inflammatory effect and can be used as a therapeutic to fight against psoriasis and other inflammatory skin diseases. Experimental \& Molecular Medicine (2017) 49, e349; doi:10.1038/emm.2017.81; published online 30 June 2017
\end{abstract}

\section{INTRODUCTION}

Psoriasis is a chronic skin inflammatory disease characterized by aberrant interaction of resident epidermal keratinocytes and effector cells, ${ }^{1-3}$ such as neutrophils, dendritic cells and activated $\mathrm{T}$ cells; its major hallmarks are abnormal proliferation and differentiation of keratinocytes. ${ }^{4,5}$ At present, the etiology of psoriasis development is not fully understood, and there is still a lack of single, cost-effective, universally accepted therapeutics. Recently, the involvement of toll-like receptors (TLRs) in the pathogenesis of psoriasis has gained wide attention. ${ }^{6}$ Therefore, the development of therapeutics that negatively modulate TLRs signaling pathways and concomitant effects in psoriasis pathogenesis is highly required. Keratinocytes are also known to express various TLRs and actively participate in the innate immune response. ${ }^{7}$ Moreover, activation of the TLR-7/nuclear factor kappa B (NF-kB) and interleukin-1 receptor-associated kinase (IRAK) pathways has been shown to increase the production of several proinflammatory mediators, such as interleukin (IL)-17, IL-23, IL-1, IL-6, and tumor necrosis factor-alpha (TNF- $\alpha$ ) that are known to amplify the inflammation. ${ }^{6-9}$ The transcription factor NF- $\mathrm{KB}$ activated by numerous inflammatory stimuli, including those sensed by TLRs has a key role in mediating the inflammatory response by regulating the expression of pro-inflammatory mediators. NF- $\mathrm{KB}$ is activated by the phosphorylation and degradation of inhibitory IкB, thereby facilitating the nuclear translocation of NF- $\mathrm{KB}^{6,}{ }^{6,7}$

Imiquimod (IMQ) is a synthetic TLR-7 agonist that is used as a topical medication against genital warts caused by human papilloma viruses. ${ }^{10}$ The clinical application of IMQ was recently reported to induce psoriasis and to exacerbate the disease in patients with well-controlled psoriasis. Topical application of IMQ has been shown to induce psoriasis-like skin inflammation through TLR-7 activation and characterized by erythema and inflammatory cell infiltration in skin. ${ }^{11,12}$ Similarly, phenotypes of the mice skin have shown the similarities with human psoriasis involving the common cytokine pathways, particularly the dependency on the IL-17/IL-23 axis. ${ }^{12-15}$

Traditionally, various natural products derived from medicinal plants have been used in the treatment of inflammatory diseases, cancers and infection. ${ }^{16-18}$ Among these natural products, rhododendrin extracted from the genus Rhododendron has been used in traditional Oriental medicine

\footnotetext{
${ }^{1}$ Department of Dermatology, Seoul St. Mary's Hospital, College of Medicine, The Catholic University of Korea, Seoul, Republic of Korea; ${ }^{2}$ Department of Dermatology, The Catholic University of Korea, St. Vincent's Hospital, Suwon, Republic of Korea and ${ }^{3}$ Natural Products Research Institute, College of Pharmacy, Seoul National University, Seoul, Republic of Korea

${ }^{4}$ These authors contributed equally to this work.

Correspondence: Professor T-Y Kim, Department of Dermatology, College of Medicine, The Catholic University of Korea, 505 Banpo-dong, Seocho-gu, Seoul 06591, Republic of Korea.

E-mail: tykimder@catholic.ac.kr

Received 1 September 2016; revised 24 January 2017; accepted 31 January 2017
} 
to treat inflammatory diseases of the skin and has been shown to have a strong anti-inflammatory effect. ${ }^{19,20}$ However, its precise mechanism of action is not clearly understood. Here, we demonstrate that rhododendrin isolated from Rhododendron brachycarpum leaves exerts significant antiinflammatory effects in IMQ-induced psoriasis-like skin inflammation in mice and investigate the basic regulatory mechanism. Our data show that rhododendrin effectively inhibits IMQ-induced skin inflammation by decreasing skin hyperplasia and the expression of inflammatory mediators such as IL-1, IL-6, IL-8, TNF- $\alpha$, IL-17, IL-23 and chemokine (c-c motif) ligand (CCL)-17 and downregulates the activation of the TLR-7/NF- $\kappa B$ and mitogen-activated protein (MAP) kinase pathways. Our results suggest that rhododendrin may be a promising and effective candidate for the treatment of inflammatory skin diseases such as psoriasis.

\section{MATERIALS AND METHODS}

\section{Isolation and purification of rhododendrin and determination of its effect on cell viability}

Rhododendrin was isolated and purified from $R$. brachycarpum using a previously described method. ${ }^{20}$ Briefly, a crude extract was prepared from powdered leaves of $R$. brachycarpum by dichloromethane and methanol treatment. The extract was then partitioned between water $\left(\mathrm{H}_{2} \mathrm{O}\right)$ and $n$-butanol, followed by partitioning in $n$-hexane and $15 \%$ aqueous methanol. An aliquot of the $15 \%$ aqueous methanol layer was subjected to reverse-phase vacuum flash chromatography and the eluents were collected as $50 \%, 40 \%, 30 \%, 20 \%, 10 \%$ aqueous methanol, $100 \%$ methanol, $100 \%$ acetone and $100 \%$ ethyl acetate. The fraction that eluted in $40 \%$ aqueous methanol was separated by semi-preparative high-performance liquid chromatography to yield rhododendrin. Final purification was achieved by reversed-phase highperformance liquid chromatography (YMC ODS-A column, $5 \mathrm{~m}$, $250 \times 4.6 \mathrm{~mm}$, YMC Europe GmBH, Dinslaken, Germany; 60\% aqueous methanol).

Cell viability was evaluated by 3-(4,5-dimethylthiaol-2-yl)-2, 5-diphenyltetrazolium bromide (MTT) reduction assay. Rhododendrintreated keratinocytes showed no toxic effects at rhododendrin concentrations of up to $300 \mu \mathrm{M}$ and exhibited more than $90 \%$ cell viability, indicating that the compound is not cytotoxic. ${ }^{20}$

\section{Mice and treatment}

Eight-week-old C57BL/6 mice were obtained from CLEA Japan (Tokyo, Japan). The mice were kept under pathogen-free conditions and provided with standard laboratory mouse chow and water. The experiments were performed in compliance with institutional guidelines and were approved by the committee of the Catholic University Institutional Animal Care and Use. Mice were shaved on the back skin and topically treated with commercially available IMQ cream (5\%) (Aldara; 3M Health Care Limited, Loughborough, UK) daily for 6 consecutive days.

To evaluate the in vivo therapeutic efficacy of rhododendrin, mice were topically treated with rhododendrin at $20 \mathrm{~mm}$ daily a few hours after IMQ application.

\section{Cell culture and reagents}

Normal human epidermal keratinocytes were procured from PromoCell (Heidelberg, Germany). The cells were maintained and cultured in keratinocyte serum-free medium supplemented with epidermal growth factor and bovine pituitary extract (Gibco BRL, Rockville, MD, USA). All experiments were performed when cells reached $\sim 70-80 \%$ confluence.

Antibodies specific for phospho- (p)-IRAK1, IRAK1, p-IRAK4, IRAK4, p-NF- $\mathrm{BB}, \mathrm{NF}-\kappa \mathrm{B}$, the phosphorylated forms of extracellular signal-regulated kinases (ERK)1/2, ERK1/2, p-p38, p38, the p-IкB kinases (IKK) $\alpha / \beta$ and glyceraldehyde-3-phosphate dehydrogenase (GAPDH) were purchased from Cell Signaling Technology (Danvers, MA, USA).

\section{Western blot analysis and immunoprecipitation}

Normal human epidermal keratinocyte cells were pre-treated with rhododendrin at various doses for $1 \mathrm{~h}$ and subsequently stimulated with $1 \mu \mathrm{g} \mathrm{ml}^{-1}$ IMQ for the indicated times. The cells were harvested, and the collected cell pellets were lysed for $30 \mathrm{~min}$ at $4{ }^{\circ} \mathrm{C}$ in ice-cold RIPA buffer containing a protease inhibitor cocktail (Roche Applied Science, Indianapolis, IN, USA). Samples containing equal amounts of protein were resolved by SDS-polyacrylamide gel electrophoresis and analyzed by immunoblotting. Briefly, membranes containing the transferred polypeptides were blocked in buffer containing 5\% skim milk in Tris-buffered saline ( $\mathrm{pH} 7.4$ ) and $0.1 \%$ Tween 20 (TBST) for $1 \mathrm{~h}$ at room temperature and subsequently probed with primary antibodies against the indicated target molecules at $4{ }^{\circ} \mathrm{C}$ overnight. The membranes were then probed with horseradish peroxidaseconjugated secondary antibodies (Invitrogen, Grand Island, NY, USA) and processed using an enhanced chemiluminescence detection kit ((iNtRON Biotechnology, Gyeonggi-do, Republic of Korea). The signals were detected on X-ray film developed in a darkroom or with an Image-Analyzer System (LAS-3000, Fujifilm, Tokyo, Japan). TRAF6 was purchased from Santa Cruz Biotechnology (Dallas, TX, USA), and immunoprecipitation was performed as previously described. ${ }^{21}$

\section{Quantitative real-time polymerase chain reaction (qPCR) analysis}

cDNA was synthesized from $1 \mu \mathrm{g}$ of total RNA using the QuantiTect Reverse Transcription Kit (Qiagen), and quantitative real-time polymerase chain reaction was performed using the KAPA SYBR fast quantitative real-time polymerase chain reaction Kit (KAPA Biosystems, Woburn, MA, USA) in a Rotor-Gene 6000 instrument (Corbett Life Science, Mortlake, NSW, Australia). The amplification program consisted of 1 cycle of $95^{\circ} \mathrm{C}$ for $10 \mathrm{~min}$ followed by 50 cycles of $95^{\circ} \mathrm{C}$ for $20 \mathrm{~s}, 55^{\circ} \mathrm{C}$ for $20 \mathrm{~s}$, and $72{ }^{\circ} \mathrm{C}$ for $20 \mathrm{~s}$ and one cycle at $72{ }^{\circ} \mathrm{C}$ for $5 \mathrm{~min}$. All the primers for interleukin (IL)- $1 \alpha$, IL- $1 \beta$, IL- 6 , IL-8, IL-23, IL-17, TNF- $\alpha$, CCL-17, and TLR-7 were purchased from Qiagen (GmbH, Hilden, Germany).

\section{Immunofluorescence staining}

Normal human epidermal keratinocyte cells were seeded at a density of $5 \times 10^{5}$ cells per well in six-well plates and incubated for $24 \mathrm{~h}$. The cells were washed with serum-free medium, pre-treated with vehicle (dimethyl sulfoxide) or rhododendrin for $1 \mathrm{~h}$ and subsequently stimulated with $1 \mu \mathrm{g} \mathrm{ml}^{-1} \mathrm{IMQ}$. The cells were then fixed in cold methanol for $10 \mathrm{~min}$, permeabilized in $0.5 \%$ Triton X-100, and blocked in PBS containing 10\% normal goat serum for $1 \mathrm{~h}$. For immunostaining, the cells were incubated with anti-NF-kB p65, TRAF6, IRAK1, TLR-7 or caveolin-1 antibodies at 1:50 dilution for $2 \mathrm{~h}$, washed three times in blocking buffer, and incubated with fluorescein isothiocyanateconjugated IgG (1:100 dilution, Molecular Probes, Eugene, OR, USA) for $1 \mathrm{~h}$. Immunofluorescence was analyzed using an inverted confocal 
a

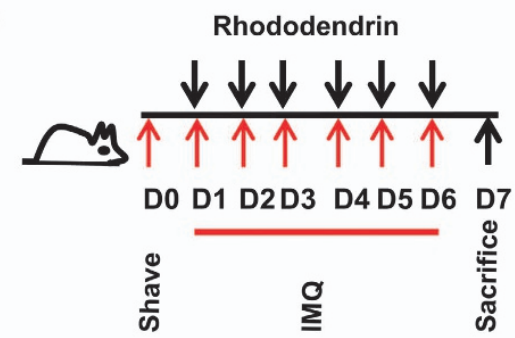

b

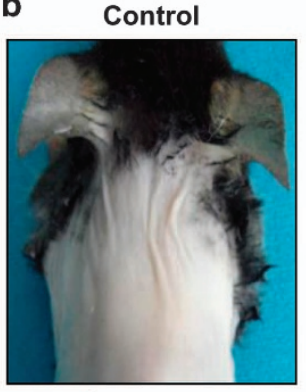

IMQ

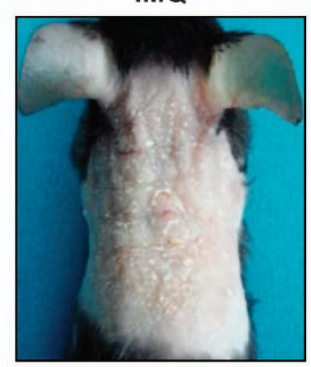

IMQ+Rhododendrin

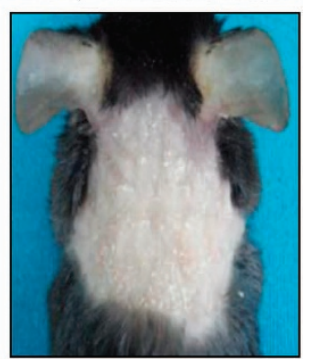

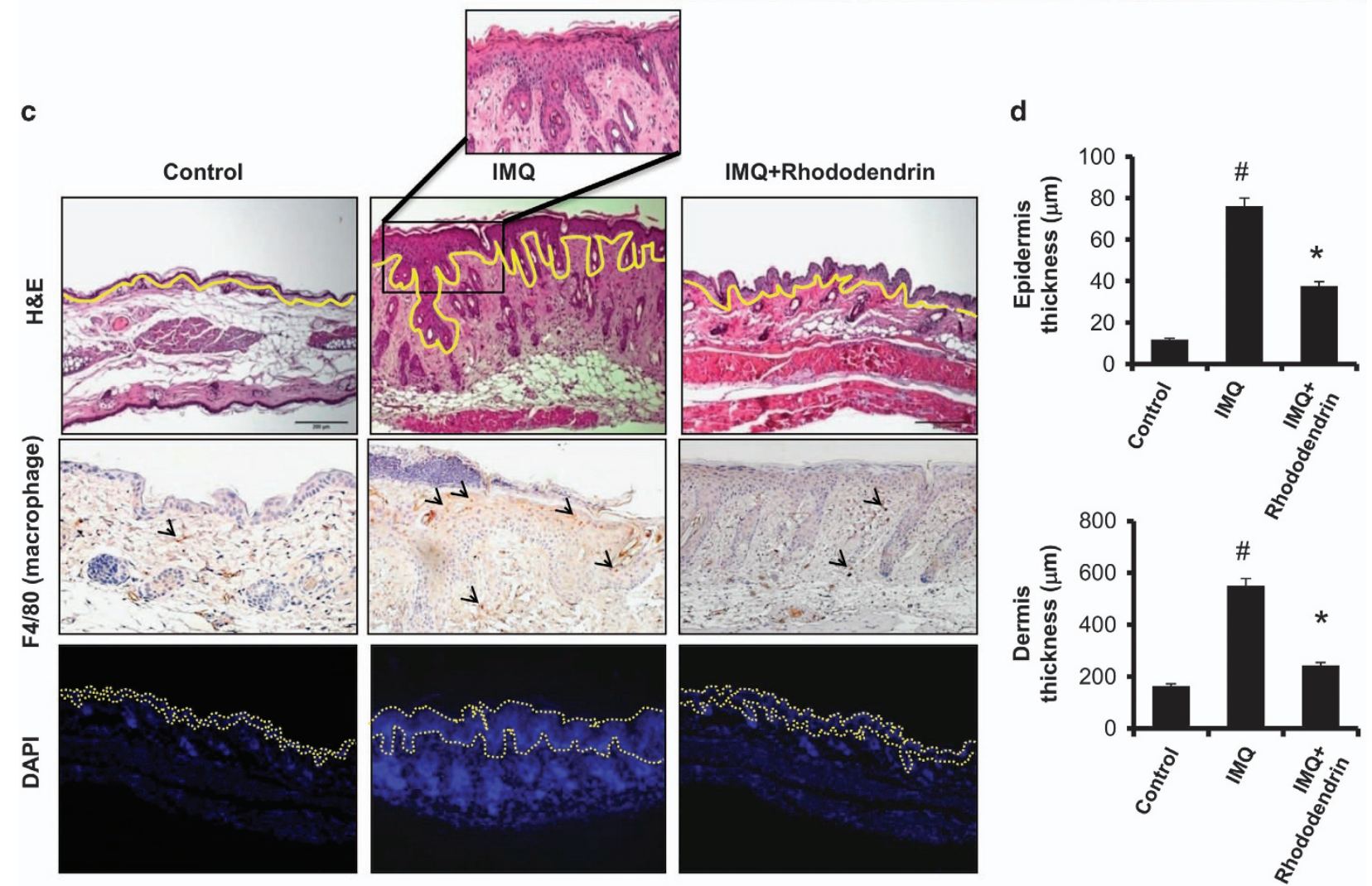

Figure 1 Rhododendrin inhibits IMQ-induced psoriasis-like inflammation. (a) In vivo experimental scheme showing the regimen of IMQ and rhododendrin treatment. (b) Phenotypical images of IMQ-induced psoriasis-like inflammation on mouse back skin with and without treatment with rhododendrin. (c) Histological assessment of back skin by H\&E staining and immunohistochemistry. (d) Epidermal and dermal thicknesses measured using an image analysis system with an Olympus microscope (Olympus). All experiments were performed at least three times. ${ }^{\#} P<0.05$ (control group versus IMQ-treated group); ${ }^{*} P<0.05$ (IMQ group versus rhododendrin and IMQ-treated group). $\mathrm{H} \& \mathrm{E}$, hematoxylin and eosin.

laser scanning microscope (Carl Zeiss, Oberkochen, Germany); nuclei were counterstained with Hoechst dye (Molecular Probes).

\section{Histological analysis and immunohistochemistry}

The ears of mice were fixed with $4 \%$ paraformaldehyde for $24 \mathrm{~h}$, washed with distilled water, dehydrated in a graded ethanol series, and embedded in paraffin. The paraffin blocks were cut into 4 - $\mu \mathrm{m}$-thick sections, mounted on glass slides, de-waxed, rehydrated in a graded series of ethanol and stained with hematoxylin and eosin. For immunohistochemical analysis, sections were incubated with the primary anti-mouse antibody F4/80 as a marker for macrophage detection and then treated with biotin-conjugated goat anti-rabbit IgG at $4{ }^{\circ} \mathrm{C}$ followed by development with $3,3^{\prime}$-diaminobenzidine (DAB).

\section{Measurement of skin thickness}

Epidermal and dermal thicknesses were measured in photomicrographs of skin sections obtained after hematoxylin and eosin staining by randomly selecting six regions using an image analysis system with an Olympus microscope (Olympus, Tokyo, Japan). The vertical thickness of the whole skin and the thickness of the epidermal layer were defined as the distance from the panniculus carnosus to the stratum corneum and as the distance from the basal layer to the stratum corneum, respectively.

\section{Statistical analysis}

The data are presented as the mean \pm s.e.m. Statistical comparisons between groups were made with the unpaired two-sided $t$-test. All experiments were performed at least three times. $P<0.05$ was considered to be statistically significant. 

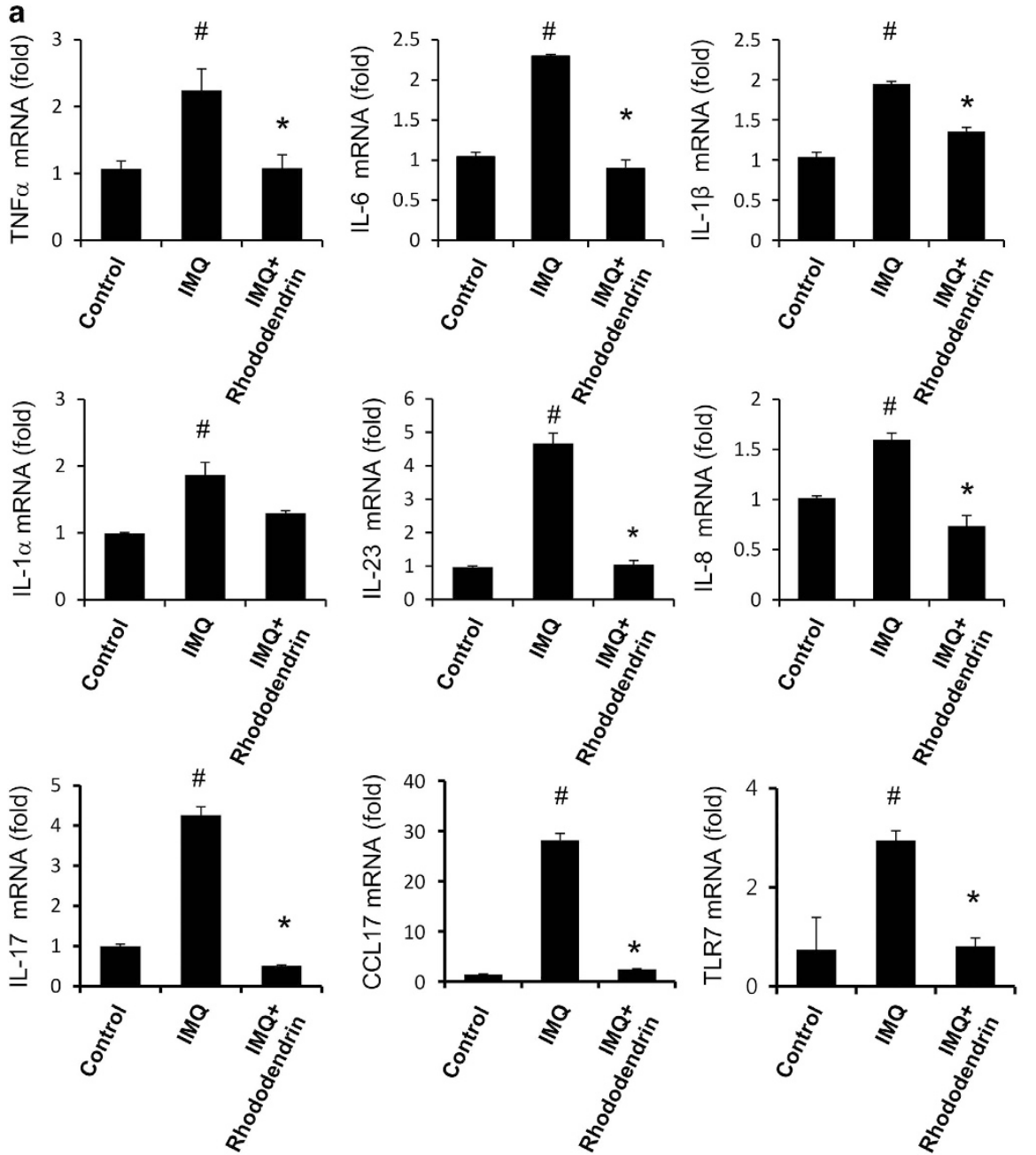

b
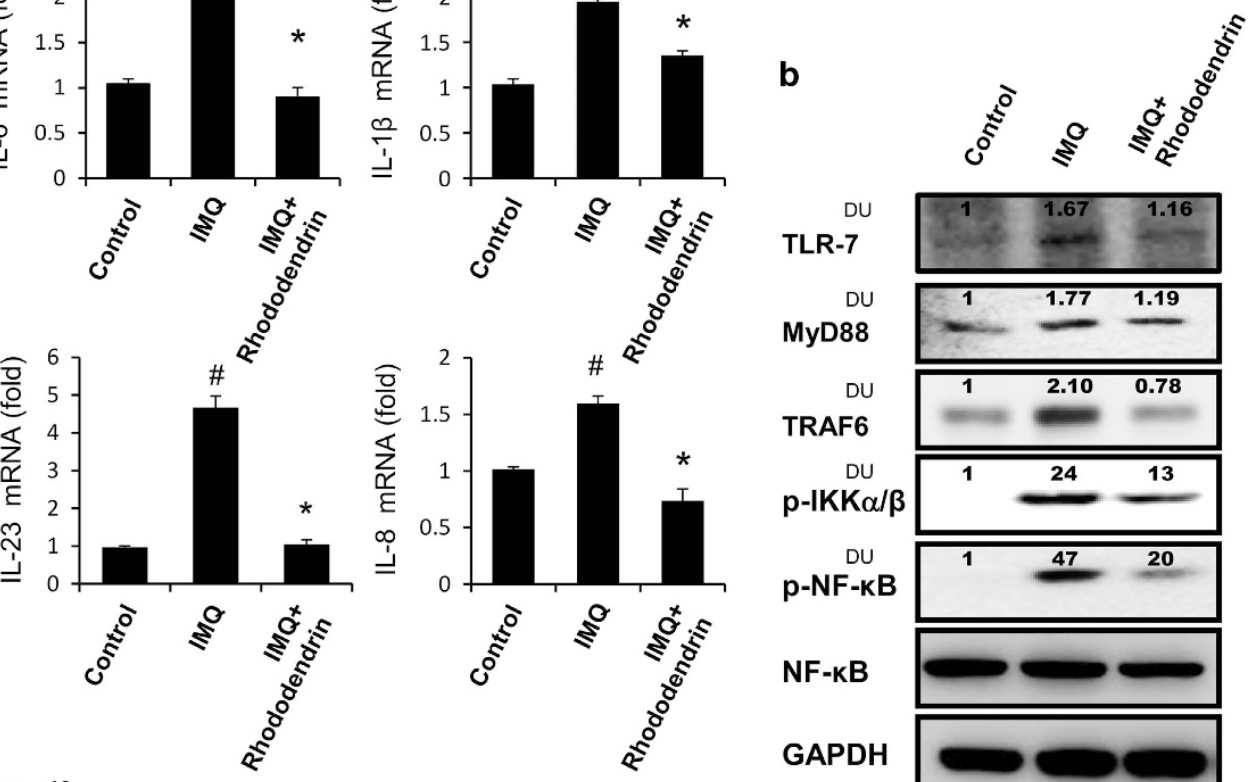

NF-KB

GAPDH
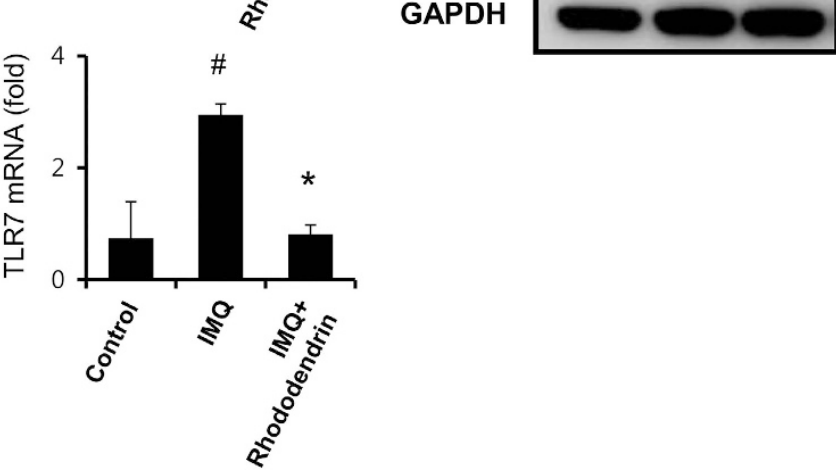

Figure 2 Rhododendrin inhibits IMQ-induced inflammatory responses in mouse skin. (a) Real-time PCR (qPCR) analysis of mRNA expression levels of pro-inflammatory mediators in mouse back skin. The graph shows the relative fold expression of mRNA normalized to GAPDH as an internal control. The data are presented as the mean \pm s.e.m. for five mice per group. ${ }^{\#} P<0.05$ (control group versus $\mathrm{IMQ}$-treated group); ${ }^{*} P<0.05$ (IMQ group versus rhododendrin and IMQ-treated group). (b) A representative western blot of mouse back skin showing TLR-7-mediated downstream events. GAPDH was used as an endogenous loading control. The numerical values shown on the blot represent mean relative DU as analyzed with ImageJ Software (National Institutes of Health, Bethesda, MD, USA). The value in the control group was set as 1 DU. DU, densitometric units; qPCR, quantitative real-time PCR.

\section{RESULTS}

Rhododendrin treatment reduces the signs and symptoms of imiquimod (IMQ)-induced psoriasis-like skin inflammation To assess whether rhododendrin treatment inhibits IMQ-induced psoriasis-like skin inflammation in mice, we applied IMQ cream on the shaved back skin of mice for six consecutive days as previously described. ${ }^{14,15}$ The mice also received rhododendrin topically following the experimental regimen (Figure 1a). Application of IMQ on the back skin increased erythema, scaling and thickening of the skin compared with the control. Interestingly, these signs and symptoms of psoriasis-like skin inflammation were effectively reduced in the rhododendrin-treated group (Figure 1b). Similarly, hematoxylin and eosin staining of mouse back skin showed that IMQ application induces hyperkeratosis, epidermal hyperplasia and increased infiltration of mononuclear inflammatory cells to the dermis, which were strongly reduced in the rhododendrin-treated mice groups (Figures 1c and d). Macrophages have a key role in inducing psoriasis-like skin disease. ${ }^{22-24}$ IMQ application increased the infiltration of the skin by macrophages, and rhododendrin treatment decreased macrophage accumulation in the skin, suggesting a protective role of rhododendrin in skin inflammation (Figure 1c, second panel).

Rhododendrin suppresses the expression of pro-inflammatory mediators and key TLR-7 downstream events in IMQ-treated mouse skin

To further evaluate the effects of rhododendrin on IMQ-induced skin inflammation in mice, we used quantitative real-time PCR to measure the gene expression levels of cytokines and chemokines that have a pivotal role in inflammation. ${ }^{15}$ Our results showed that rhododendrin treatment decreased the IMQ-induced mRNA expression of several pro-inflammatory 
a
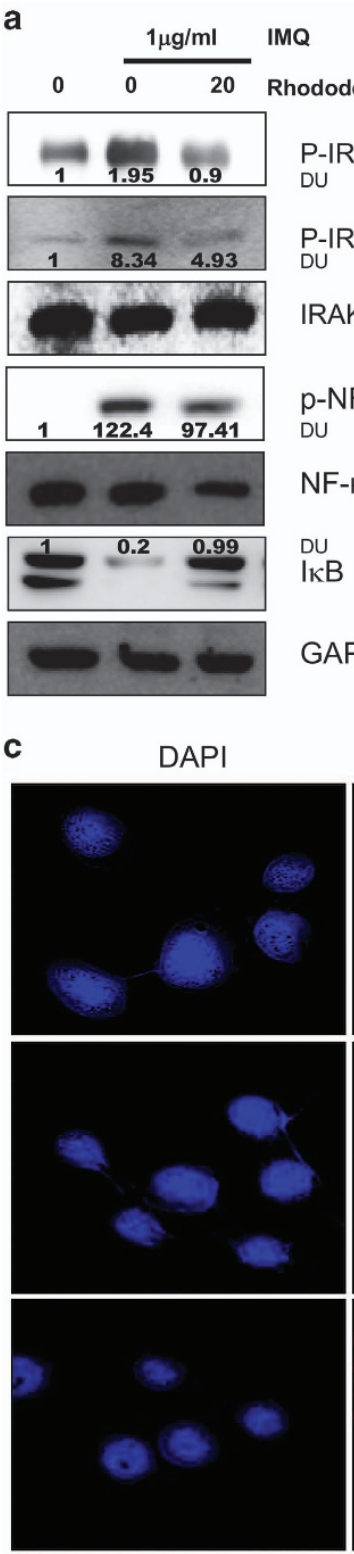

b

\begin{tabular}{|c|c|c|c|c|c|}
\hline \multirow[b]{2}{*}{0} & \multicolumn{4}{|c|}{$1 \mu \mathrm{g} / \mathrm{ml}$} & \multirow{2}{*}{$\begin{array}{l}\text { IMQ } \\
\text { Rhododendrin }(\mu \mathrm{M})\end{array}$} \\
\hline & 0 & 5 & 10 & 20 & \\
\hline 1 & 13.4 & 15.16 & 7.35 & 6.6 & $\begin{array}{l}\text { DU } \\
\text { pMEK }\end{array}$ \\
\hline
\end{tabular}

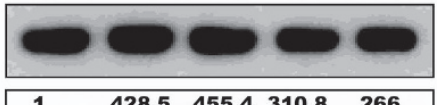

MEK

DU

p-p38

p38

DU

COX-2

GAPDH

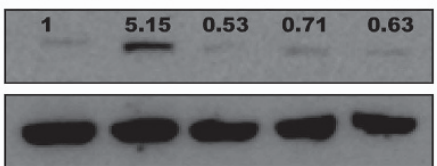

GAPDH
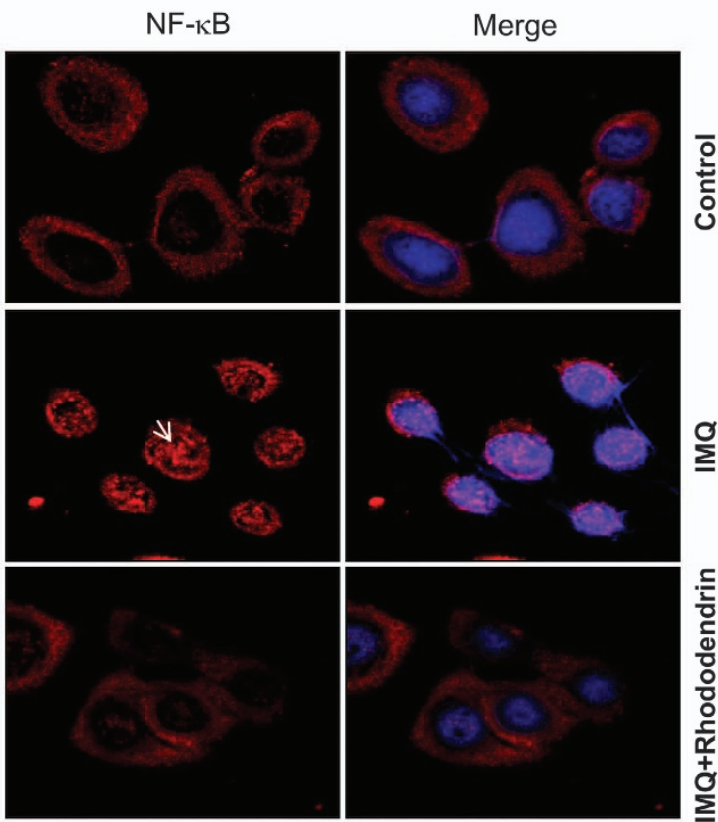

Figure 3 Rhododendrin inhibits IMQ-induced TLR-7-mediated downstream signaling and MAP kinase pathways in NHEK. (a, b) NHEK cells were pre-treated with rhododendrin for $1 \mathrm{~h}$ and stimulated with IMQ for $15 \mathrm{~min}$, and western blot analysis was performed with the indicated antibodies. The data shown are representative of three independent experiments. GAPDH served as a loading control. The numerical values shown on the blot represent mean relative DU as analyzed with ImageJ Software (National Institutes of Health, USA). The value in the untreated control was set as $1 \mathrm{DU}$. (c) Rhododendrin inhibited the IMQ-induced translocation of NF- $\mathrm{KB}$ into the nucleus as evaluated by inverted fluorescence microscopy. Rhododendrin, $20 \mu \mathrm{M} ; \mathrm{IMQ}, 1 \mu \mathrm{g} \mathrm{ml} \mathrm{I}^{-1}$. DU, densitometric units; NHEK, normal human epidermal keratinocytes.

mediators, including IL-1, IL-6, IL-8, IL-17, IL-23, TNF- $\alpha$ and CCL-17 in the back skin of the treated mice. In addition, the level of TLR-7 mRNA was found to be upregulated in IMQtreated mice, and this was effectively reduced by rhododendrin treatment (Figure 2a). Similarly, TLR-7 activation at the protein level and key downstream events such as the activation of myeloid differentiation primary response protein 88 (MyD88), TNF-receptor-associated factor 6 (TRAF6), IKK and NF- $\mathrm{BB}$ were markedly reduced in the rhododendrin-treated group compared with the group treated with IMQ alone (Figure $2 \mathrm{~b}$ ).
Rhododendrin treatment decreases the level of IMQ-induced TLR-7 downstream targets and MAP kinase signaling in normal human epidermal keratinocytes

IMQ has been reported to exert its biological activity through TLR-7 activation. ${ }^{15,23}$ Moreover, previous reports have shown that TLR-7 is expressed and highly upregulated in cultured keratinocytes upon IMQ treatment, thus potentiating the inflammatory response in the skin. 8,9

To investigate whether rhododendrin regulates IMQinduced TLR-7 downstream events in cultured keratinocytes, 

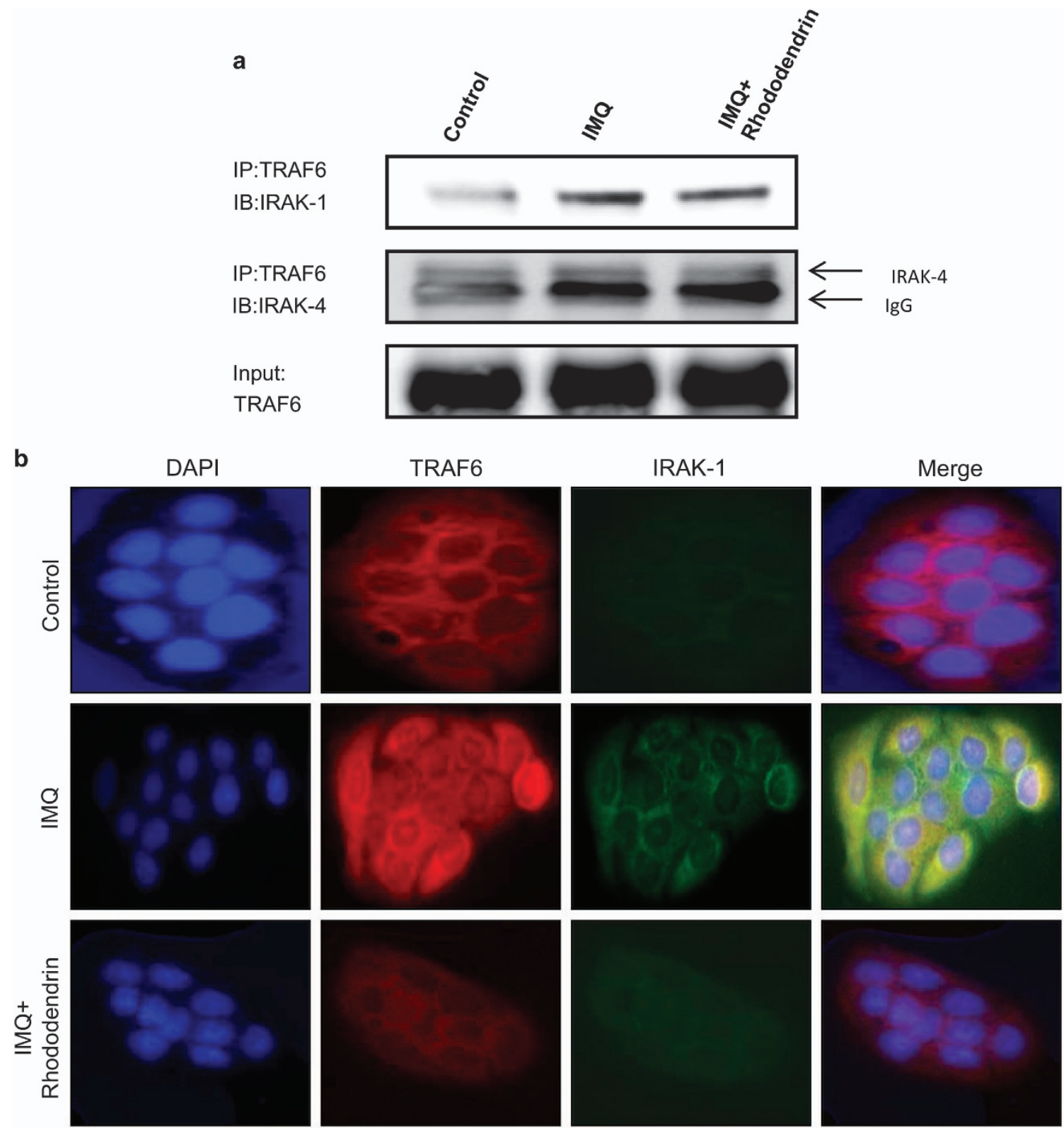

Figure 4 Rhododendrin suppresses the interaction of TRAF6 and IRAK1 that is necessary for TLR-7 activation. (a) NHEK lysates were immunoprecipitated with anti-TRAF6 antibodies, and detected by IRAK1 and IRAK4 through western blotting. (b) Immunocytochemical analysis of NHEKs that were stained with TRAF6 antibody (red), IRAK1 antibody (green), and DAPI for nuclear staining (blue) following treatment of the cells with IMQ alone $\left(1 \mu \mathrm{g} \mathrm{ml^{-1 }}\right)$ or with IMQ and rhododendrin $(20 \mu \mathrm{M})$.

we pre-treated the cells with rhododendrin and induced the TLR-7 activation cascade by exposing the cells to IMQ. Interestingly, our data showed that rhododendrin significantly downregulated the activated TLR-7 key downstream events such as IRAK1/4 and NF- $\mathrm{KB}$ and prevented the degradation of I $\mathrm{KB}$ in keratinocytes (Figure $3 \mathrm{a}$ ). We also examined the effects of rhododendrin on the MAP kinase pathway and on cyclooxygenase-2 (COX-2) activation, both of which are known to have a critical role in skin inflammation. ${ }^{24-30}$ As shown in Figure 3b, rhododendrin treatment effectively decreased the phosphorylation of MEK and p38 and the activation of COX-2 compared with IMQ alone. Moreover, rhododendrin treatment inhibited the translocation of the key inflammatory transcription factor NF- $\mathrm{KB}$ into the nucleus (Figure 3c), further suggesting an anti-inflammatory role of rhododendrin in the regulation of psoriasis pathogenesis.

\section{Rhododendrin significantly inhibits the IMQ-induced interaction of IRAK1 with TRAF6 in cultured primary keratinocytes}

TRAF6 is known to interact with IRAK and regulates several signaling cascades involved in the immune response and in the maintenance of homeostasis. ${ }^{31-35}$ As we demonstrated above, IMQ treatment induces TRAF6 and IRAK-1/4 expression at the 


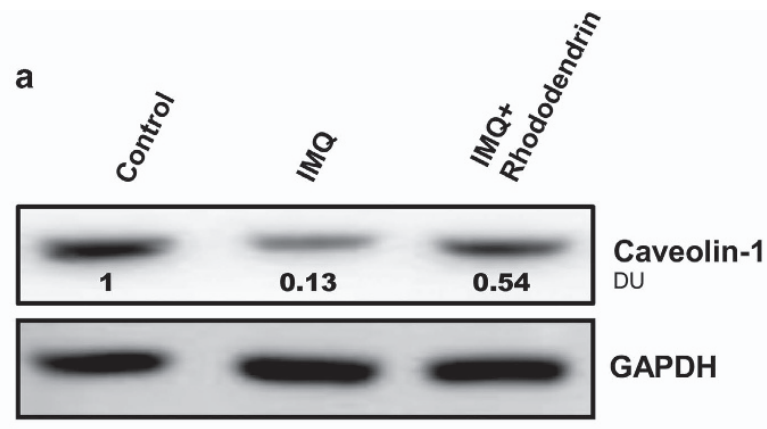

b
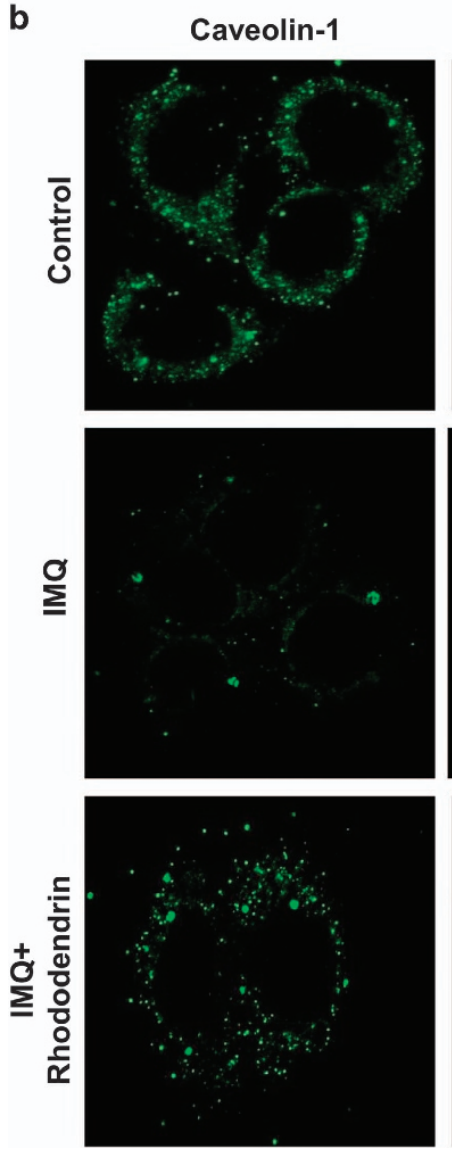

TLR-7
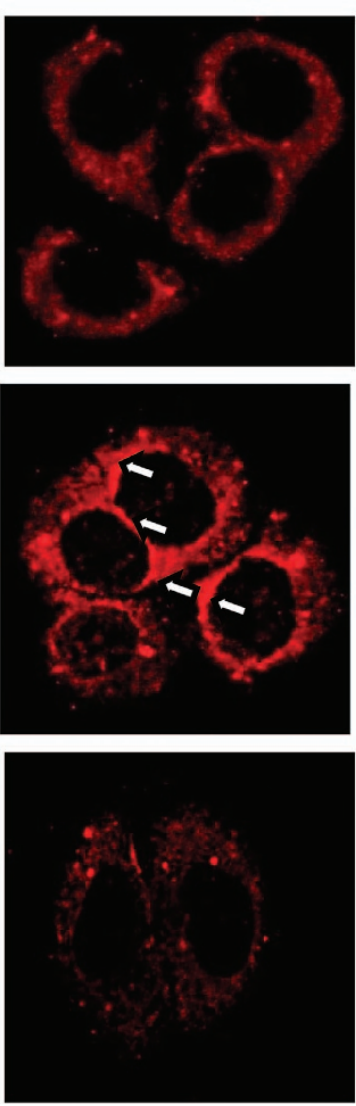

DAPI
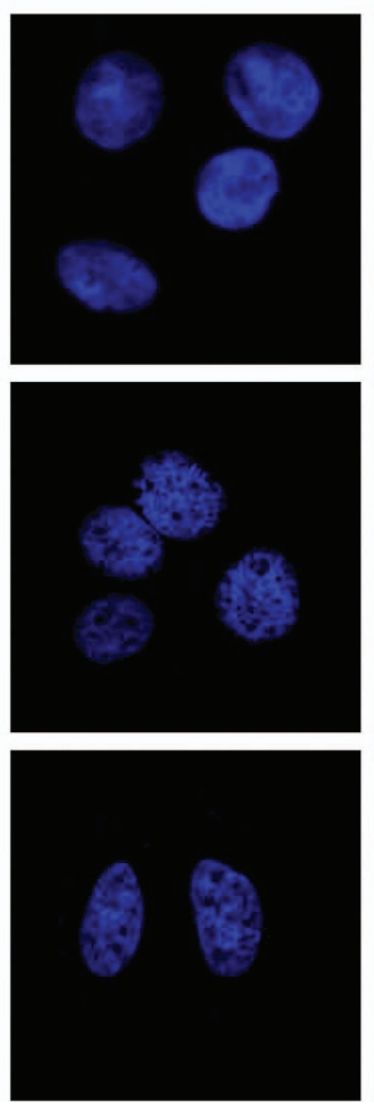
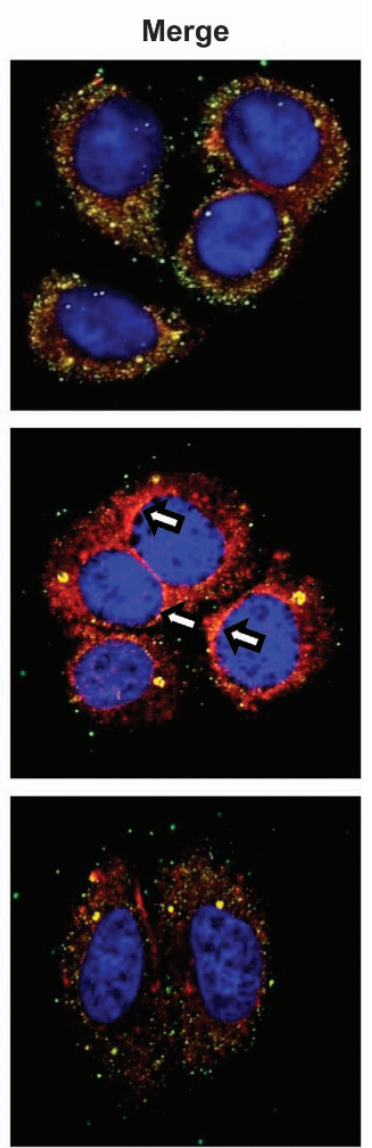

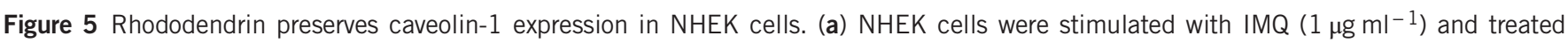
with rhododendrin at $20 \mu \mathrm{m}$ for $1 \mathrm{~h}$, and caveolin-1 expression at the protein level was evaluated by immunoblotting. The numerical values shown on the blot represent mean relative DU as analyzed with ImageJ Software (National Institutes of Health, USA). The value obtained for the untreated control was set as 1 DU. (b) NHEK cells were also stained for TLR-7 and caveolin-1 to evaluate their expression and their association at the protein level by immunocytochemical analysis. DU, densitometric units.

protein level in skin and in keratinocytes which was decreased in the presence of rhododendrin. Interestingly, rhododendrin also inhibited the IMQ-induced interaction of TRAF6 and IRAK1, as evaluated by immunoprecipitation assays (Figures $4 \mathrm{a}$ and $\mathrm{b}$ ).

\section{Rhododendrin preserves caveolin-1 expression in} IMQ-treated keratinocytes

Caveolin-1 is involved in the proliferation and differentiation of skin cells and in the maintenance of homeostasis in skin. Loss of caveolin-1 in epidermal keratinocytes has been shown to contribute to the development of psoriasis. ${ }^{36-39}$ Therefore, we evaluated whether rhododendrin also regulates caveolin-1 expression. Surprisingly, caveolin-1 expression was found to be preserved with downregulation of TLR-7 upon treatment with rhododendrin compared with keratinocytes treated with IMQ alone, suggesting that rhododendrin exerts a critical therapeutic action in the maintenance of skin homeostasis during disease pathogenesis (Figures 5a and $\mathrm{b}$ ).

\section{DISCUSSION}

TLRs have been reported to be involved in the pathogenesis of several autoimmune diseases, including psoriasis and 
rheumatoid arthritis. ${ }^{40,41}$ In general, TLR-7 activation leads to the induction of the NF- $\kappa \mathrm{B}$ pathway through assembly of the MyD88, IRAK and TRAF6 complex and activates the inflammatory response. ${ }^{41}$ Keratinocytes and effector cells such as macrophages and dendritic cells are also known to express high levels of TLR-7 receptors and activate inflammatory cascades in response to aberrant environments, resulting in the production of several pro-inflammatory mediators such as IL-1, IL-17, IL-23 and TNF- $\alpha$, which have crucial roles in the amplification and progression of inflammatory disease. ${ }^{42}$

Psoriasis is a chronic, inflammatory, autoimmune skin disease that has been reported to be associated with a self-amplifying loop of TLR-7 that contributes to the disease pathogenesis. $3,15,23,43$ Therefore, effective therapeutic value targeting these receptors and/or TLRs-mediated key events in controlling the psoriasis-like skin inflammation is highly on demand. To explore potential therapeutic drugs for psoriasis in an IMQ-induced mouse model of psoriasis, we sought to investigate the effect of the naturally occurring phytochemical rhododendrin. Rhododendrin, a natural compound that is present in abundance in the leaves of plants of the genus Rhododendron, is known to have anti-oxidant, anti-cancer, anti-viral and anti-inflammatory activity. ${ }^{19,20}$ In previous work, we showed that rhododendrin can regulate skin inflammation through effective inhibition of NF- $\mathrm{BB}, \mathrm{MAPK}$ and PI3K/AKT signaling. ${ }^{20}$ However, study of the effects of rhododendrin in psoriasis-like skin inflammation is still in its infancy. Here, we demonstrated that rhododendrin can inhibit IMQ-induced psoriasis-like inflammatory responses both in vivo and in vitro, probably by reducing the infiltration of inflammatory cells in the vicinity of the affected skin, regulating activation of the TLR-7/NF- $\mathrm{KB}$ axis, and inhibiting the production of a number of pro-inflammatory mediators. Among the various proinflammatory mediators, IL-17 and IL-23 cytokines have been postulated to drive the development of Th17 cells, which are known to be the major cell type involved in the pathogenesis of psoriasis. The production of these cytokines further promotes Th17 cell maturation and results in erythema, a mixed inflammatory cell infiltrates and hyperplasia. ${ }^{12,15,23}$ Our data show that rhododendrin effectively suppresses IL-17 and IL-23 expression at the mRNA level, demonstrating its possible role in controlling Th17 cell development and hence its potential efficacy in preventing the Th17-driven pathogenesis of psoriasis and other inflammatory diseases such as atopic dermatitis, rheumatoid arthritis, inflammatory bowel disease and colitis.

Activation of the ERK and p38 MAP kinase pathways has been reported to be increased in psoriatic skin lesions compared with non-lesional psoriatic skin. ${ }^{25-27}$ Consistent with these results, we also found increased activation of MAP kinases in mouse back skin and cultured keratinocytes treated with IMQ. The activation of MAP kinases was completely inhibited by treatment with rhododendrin, suggesting several possible molecular mechanisms of rhododendrin action in the resolution of inflammation. Our data also showed that rhododendrin inhibits TLR-7 activation by disrupting the interaction of MyD88 and TRAF6, which form a complex that is required for initiation of the inflammatory response. However, it is also possible that rhododendrin could regulate direct trafficking of TLR-7 from endoplasmic reticulum to endosomes through proteasome-degrading activity of accessory proteins that are required to escort TLRs or by preventing the endosomal acidification that is required for TLR-7 activation. Further investigation aimed at understanding the molecular targets of rhododendrin is certainly warranted.

Caveolin-1 is a membrane protein that is required for signal transduction, cellular trafficking and maintenance of membrane cholesterol homeostasis. It has been shown that the expression level of caveolin-1 is strikingly decreased and its spatial pattern of expression is altered in psoriatic skin. ${ }^{36-39}$ Considerable work has been done to correlate caveolin-1 expression and the activity of receptor kinases with their respective downstream effects. ${ }^{44}$ Activation of receptor tyrosine kinases and of the downstream p42/p44 MAP kinase pathway has been implicated in the keratinocyte hyperproliferation that is commonly associated with the pathogenesis of psoriasis. $27,39,45,46$ Interestingly, our results showed that rhododendrin preserves caveolin-1 expression at the protein level by preventing its decrease upon IMQ treatment, suggesting its role in maintaining membrane integrity and skin homeostasis.

In conclusion, our data strongly suggest that rhododendrin targets multiple molecules to inhibit TLR-7 activation and could be a distinct from known therapeutics in reducing the psoriasis-like skin inflammation and other inflammatory conditions through its antioxidant and anti-inflammatory properties.

\section{CONFLICT OF INTEREST}

The authors declare no conflict of interest.

\section{ACKNOWLEDGEMENTS}

This study was supported by a grant from the BioGreen 21 Program (PJ007175) of the Rural Development Administration, Republic of Korea.

1 Cai Y, Fleming C, Yan J. New insights of T cells in the pathogenesis of psoriasis. Cell Mol Immunol 2012; 9: 302-309.

2 Lowes MA, Bowcock AM, Krueger JG. Pathogenesis and therapy of psoriasis. Nature 2007; 445: 866-873.

3 Nestle FO, Kaplan DH, Barker J. Psoriasis. N Engl J Med 2009; 361: 496-509.

4 Krueger JG. Hiding under the skin: a welcome surprise in psoriasis. Nat Med 2012; 18: 1750-1751.

5 Schon MP, Boehncke WH. Psoriasis. N Engl J Med 2005; 352 : 1899-1912.

6 Hari A, Flach TL, Shi Y, Mydlarski PR. Toll-like receptors: role in dermatological disease. Mediators Inflamm 2010; 2010: 437246.

7 Miller LS, Modlin RL. Toll-like receptors in the skin. Semin Immunopathol 2007; 29: 15-26.

8 Sun J, Zhao Y, Hu J. Curcumin inhibits imiquimod-induced psoriasis-like inflammation by inhibiting IL-1beta and IL-6 production in mice. PLoS ONE 2013; 8: e67078.

9 Li ZJ, Sohn KC, Choi DK, Shi G, Hong D, Lee HE et al. Roles of TLR7 in activation of NF-kappaB signaling of keratinocytes by imiquimod. PLoS ONE 2013; 8: e77159. 
10 Bong AB, Bonnekoh B, Franke I, Schon M, Ulrich J, Gollnick H. Imiquimod, a topical immune response modifier, in the treatment of cutaneous metastases of malignant melanoma. Dermatology 2002; 205: 135-138.

11 Stary G, Bangert C, Tauber M, Strohal R, Kopp T, Stingl G. Tumoricida activity of TLR7/8-activated inflammatory dendritic cells. J Exp Med 2007; 204: 1441-1451.

12 El Malki K, Karbach SH, Huppert J, Zayoud M, Reissig S, Schüler R et al. An alternative pathway of imiquimod-induced psoriasis-like skin inflammation in the absence of interleukin-17 receptor a signaling. J Invest Dermatol 2013; 133: 441-451.

13 Shibata S, Tada Y, Asano Y, Yanaba K, Sugaya M, Kadono T et al. IL-27 activates Th1-mediated responses in imiquimod-induced psoriasis-like skin lesions. J Invest Dermatol 2013; 133: 479-488.

14 Van Belle AB, De Heusch M, Lemaire MM, Hendrickx E, Warnier G, Dunussi-Joannopoulos $\mathrm{K}$ et al. IL-22 is required for imiquimod-induced psoriasiform skin inflammation in mice. J Immunol 2012; 188: 462-469.

15 Van der Fits L, Mourits S, Voerman JS, Kant M, Boon L, Laman JD et al. Imiquimod-induced psoriasis-like skin inflammation in mice is mediated via the IL-23/IL-17 axis. J Immunol 2009; 182: 5836-5845.

16 Yin SY, Wei WC, Jian FY, Yang NS. Therapeutic applications of herbal medicines for cancer patients. Evid Based Complement Alternat Med 2013; 2013: 302-426.

17 Ghasemian M, Owlia S, Owlia MB. Review of anti-inflammatory herbal medicines. Adv Pharmacol Sci 2016; 2016: 9130979.

$18 \mathrm{Kim} \mathrm{BH}, \mathrm{Oh} \mathrm{I}, \mathrm{Kim} \mathrm{JH}$, Jeon JE, Jeon B, Shin J et al. Anti-inflammatory activity of compounds isolated from Astragalussinicus $L$. in cytokineinduced keratinocytes and skin. Exp Mol Med 2014; 46: e87.

$19 \mathrm{Kim} \mathrm{MH}$, Nugroho A, Choi J, Park JH, Park HJ. Rhododendrin, an analgesic/anti-inflammatory arylbutanoid glycoside, from the leaves of Rhododendron aureum. Arch Pharm Res 2011; 34: 971-978.

20 Jeon YJ, Kim BH, Kim S, Oh I, Lee S, Shin J et al. Rhododendrin ameliorates skin inflammation through inhibition of NF-kB, MAPK, and PI3K/Akt signaling. Eur J Pharmacol 2013; 714: 7-14.

21 Kaboord B, Perr M. Isolation of proteins and protein complexes by immunoprecipitation. Methods Mol Biol 2008; 424: 349-364.

22 Clark RA, Kupper TS. Misbehaving macrophages in the pathogenesis of psoriasis. J Clin Invest 2006; 116: 2084-2087.

23 Sah SK, Park KH, Yun CO, Kang KS, Kim TY. Effects of human mesenchymal stem cells transduced with superoxide dismutase on imiquimod-induced psoriasis-like skin inflammation in mice. Antioxid Redox Signal 2016; 24: 233-248.

24 De Meyer I, Martinet W, Schrijvers DM, Timmermans JP, Bult H, De Meyer GR. Toll-like receptor 7 stimulation by imiquimod induces macrophage autophagy and inflammation in atherosclerotic plaques. Basic Res Cardiol 2012; 107: 269.

25 Johansen C, Kragballe K, Westergaard M, Henningsen J, Kristiansen K, Iversen $\mathrm{L}$. The mitogen-activated protein kinases p38 and ERK $1 / 2$ are increased in lesional psoriatic skin. Br J Dermatol 2005; 152: 37-42.

26 Mose M, Kang Z, Raaby L, Iversen L, Johansen C. TNFalpha- and IL-17A-mediated S100A8 expression is regulated by p38 MAPK. Exp Dermatol 2013; 22: 476-481.

27 Haase I, Hobbs RM, Romero MR, Broad S, Watt FM. A role for mitogen-activated protein kinase activation by integrins in the pathogenesis of psoriasis. J Clin Invest 2001; 108: 527-536.

28 Dimon-Gadal S, Raynaud F, Evain-Brion D, Keryer G. MAP kinase abnormalities in hyperproliferative cultured fibroblasts from psoriatic skin. J Invest Dermatol 1998; 110: 872-879.

29 Bakry OA, Samaka RM, Shoeib MA, Abdel Aal SM. Nuclear factor kappa B and cyclo-oxygenase-2: two concordant players in psoriasis pathogenesis. Ultrastruct Pathol 2015; 39: 49-61.
30 Cho JW, Park K, Kweon GR, Jang BC, Baek WK, Suh MH et al. Curcumin inhibits the expression of COX-2 in UVB-irradiated human keratinocytes (HaCaT) by inhibiting activation of AP-1: p38 MAP kinase and JNK as potential upstream targets. Exp Mol Med 2005; 37: $186-192$

$31 \mathrm{Kim}$ JE, Kim SY, Lim SY, Kieff E, Song YJ. Role of $\mathrm{Ca}^{2+} /$ calmodulindependent kinase II-IRAK1 interaction in LMP1-induced NF-kappaB activation. Mol Cell Biol 2014; 34: 325-334.

32 Hemmi H, Kaisho T, Takeuchi O, Sato S, Sanjo H, Hoshino K et al. Small anti-viral compounds activate immune cells via the TLR7 MyD88-dependent signaling pathway. Nat Immunol 2002; 3: 196-200.

33 Kawai T, Akira S. TLR signaling. Cell Death Differ 2006; 13: 816-825.

34 Moresco EM, LaVine D, Beutler B. Toll-like receptors. Cur Biol 2011; 21: R488-R493.

35 O'Neill LA, Bowie AG. The family of five: TIR-domain-containing adaptors in Toll-like receptor signalling. Nat Rev Immunol 2007; 7: 353-364.

36 Zhang F, Li H, Zhou Y, Gu Y, Wang L. Caveolin-1 expression in different types of psoriatic lesions: analysis of 66 cases. Indian J Dermatol 2014; 59: 225-229.

37 Campbell L, Laidler P, Watson RE, Kirby B, Griffiths CE, Gumbleton M. Downregulation and altered spatial pattern of caveolin-1 in chronic plaque psoriasis. Br J Dermatol 2002; 147: 701-709.

38 Ma WY, Zhuang L, Cai DX, Zhong H, Zhao C, Sun Q. Inverse correlation between caveolin-1 expression and clinical severity in psoriasis vulgaris. J Int Med Res 2012; 40: 1745-1751.

39 Fine SW, Lisanti MP, Galbiati F, Li M. Elevated expression of caveolin-1 in adenocarcinoma of the colon. Am J Clin Pathol 2001; 115: 719-724.

40 Medzhitov R. Recognition of microorganisms and activation of the immune response. Nature 2007; 449: 819-826.

41 Akira S, Takeda K. Toll-like receptor signalling. Nat Rev Immunol 2004; 4 : 499-511.

42 Zarember KA, Godowski PJ. Tissue expression of human Toll-like receptors and differential regulation of Toll-like receptor mrnas in leukocytes in response to microbes, their products, and cytokines. J Immunol 2002; 168: 554-561.

43 Lai CY, Yeh DW, Lu CH, Liu YL, Huang LR, Kao CY et al. Identification of thiostrepton as a novel inhibitor for psoriasis-like inflammation induced by TLR7-9. J Immunol 2015; 195: 3912-3921.

44 Zundel W, Swiersz LM, Giaccia A. Caveolin 1-mediated regulation of receptor tyrosine kinase-associated phosphatidylinositol 3-kinase activity by ceramide. Mol Cell Biol 2000; 20: 1507-1514.

45 Wrone-Smith T, Mitra RS, Thompson CB, Jasty R, Castle VP, Nickoloff BJ. Keratinocytes derived from psoriatic plaques are resistant to apoptosis compared with normal skin. Am J Pathol 1997; 151: 1321-1329.

46 Nanney LB, Yates RA, King LE Jr. Modulation of epidermal growth factor receptors in psoriatic lesions during treatment with topical EGF. J Invest Dermatol 1992; 98: 296-301.

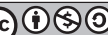

This work is licensed under a Creative Commons Attribution-NonCommercial-ShareAlike 4.0 International License. The images or other third party material in this article are included in the article's Creative Commons license, unless indicated otherwise in the credit line; if the material is not included under the Creative Commons license, users will need to obtain permission from the license holder to reproduce the material. To view a copy of this license, visit http://creativecommons.org/licenses/by-nc-sa/4.0/ 